\title{
BMJ Open Following young people with perinatal HIV infection from adolescence into adulthood: the protocol for PHACS AMP Up, a prospective cohort study
}

Katherine Tassiopoulos, ${ }^{1}$ Kunjal Patel, ${ }^{1}$ Julie Alperen, ${ }^{1}$ Deborah Kacanek, ${ }^{2}$ Angela Ellis, ${ }^{3}$ Claire Berman, ${ }^{1}$ Susannah M Allison, ${ }^{4}$ Rohan Hazra, ${ }^{5}$ Emily Barr, ${ }^{6}$ Krystal Cantos, ${ }^{1}$ Suzanne Siminski, ${ }^{3}$ Michael Massagli, ${ }^{1}$ Jose Bauermeister, ${ }^{7}$ Danish Q Siddiqui, ${ }^{8}$ Ana Puga, ${ }^{9}$ Russell Van Dyke, ${ }^{8}$ George R Seage III, ${ }^{1}$ for the Pediatric HIV/AIDS Cohort Study

To cite: Tassiopoulos $\mathrm{K}$, Patel K, Alperen J, et al. Following young people with perinatal HIV infection from adolescence into adulthood: the protocol for PHACS AMP Up, a prospective cohort study. BMJ Open 2016;6: e011396. doi:10.1136/ bmjopen-2016-011396

- Prepublication history for this paper is available online. To view these files please visit the journal online (http://dx.doi.org/10.1136/ bmjopen-2016-011396).

Received 3 February 2016 Revised 3 May 2016 Accepted 4 May 2016

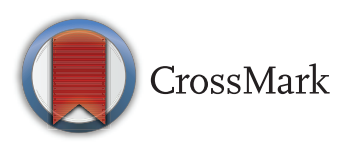

For numbered affiliations see end of article.

Correspondence to Dr Katherine Tassiopoulos; ktassiop@hsph.harvard.edu

\section{ABSTRACT}

Introduction: The first generation of adolescents born with HIV infection has reached young adulthood due to advances in treatment. It is important to continue follow-up of these individuals to assess their long-term medical, behavioural and mental health and ability to successfully transition to adulthood while coping with a chronic, potentially stigmatising condition. To accomplish this, and to maintain their interest in longterm research participation, we need to accommodate the changing lifestyles and interests of young adult study participants while ensuring valid data collection. We report the protocol for Pediatric HIV/AIDS Cohort Study (PHACS) Adolescent Master Protocol (AMP) Up, a prospective cohort study enrolling young adult participants for long-term follow-up.

Methods and analysis: AMP Up is recruiting 850 young men and women 18 years of age and older600 perinatally HIV-infected and a comparison group of 250 perinatally HIV-exposed, uninfected-at 14 clinical research sites in the USA and Puerto Rico. Recruitment began in April 2014 and is ongoing, with 305 participants currently enrolled. Planned follow-up is $\geq 6$ years. Data are collected with a flexible hybrid of online and in-person methods. Outcomes include: transition to adult clinical care and retention in care; end-organ diseases; malignancies; metabolic complications; sexually transmitted infections; reproductive health; mental health and neurocognitive functioning; adherence to antiretroviral treatment; sexual behaviour and substance use; hearing and language impairments; and employment and educational achievement.

Ethics and dissemination: The study received ethical approval from the Harvard T.H. Chan School of Public Health's institutional review board (IRB), and from the IRBs of each clinical research site. All participants provide written informed consent; for cognitively impaired individuals with legally authorised representatives, legal guardian permission and participant assent is obtained. Findings will be disseminated through peer-reviewed journals, conference presentations and participant summaries.

\section{Strengths and limitations of this study}

- The Pediatric HIV/AIDS Cohort Study (PHACS) Adolescent Master Protocol (AMP) Up study will enable long-term follow-up of perinatally HIV-infected $(\mathrm{PHIV}+)$ young adults for evaluation of a wide range of clinical, behavioural and mental health outcomes, as well as transition to adult clinical care and adult functioning.

- This study design allows flexibility in the transition from in-person to remote data collection, and can be adopted by other prospective epidemiological studies concerned with optimising participant retention over the long term.

- Since AMP Up is enrolling young adults through the PHACS research clinical sites, participants may be more likely to be adherent to clinical care and supportive services; young adults nonadherent or lost to care may not be well represented.

\section{INTRODUCTION}

The first generation of youth perinatallyinfected with HIV (PHIV+) has reached late adolescence and young adulthood. The Pediatric HIV/AIDS Cohort Study (PHACS), the largest long-term study of perinatal HIV infection in the USA, has advanced understanding of the effects of antiretroviral medications (ARVs) and HIV infection on infectious and non-infectious complications, and metabolic, growth, cardiovascular, neurodevelopmental, neurological and behavioural outcomes among youth and adolescents. ${ }^{1-17}$ Continued follow-up into young adulthood is needed in order to assess the longer term effects of HIV and ARVs on these outcomes, ${ }^{18} 19$ and to examine issues specific to these emerging young adults, including the effects of transitioning from paediatric to adult healthcare on clinical and behavioural 
outcomes and retention in care, the attainment of milestones of adulthood including higher education and employment, reproductive outcomes and the risks of HIV transmission to sexual partners and offspring.

The PHACS Adolescent Master Protocol (AMP) is a prospective cohort study that enrolled youth with PHIV+ and a comparison cohort of perinatally HIV exposed, uninfected youth (PHEU) who were 7-16 years old at enrolment. Participants are followed at 14 research clinic sites across the USA including Puerto Rico. The first AMP participants reached 18 years of age in 2009. While overall study retention remains strong, we found that older AMP participants were being disproportionately lost to follow-up. The AMP Up protocol was therefore conceived as a protocol tailored specifically to young adults, in order to maximise validity of data collection as well as retention of participants in continued long-term follow-up.

Development of novel strategies to optimise sustained research participation is a critical challenge for all longterm epidemiological studies. ${ }^{20} 21$ For young adults involved in longitudinal studies since childhood, participation may become less appealing as they gain independence, secure employment or attend college, and thereby become less willing or able to return to clinics for study visits. An additional disincentive exists if the participant does not have a personal connection with the study and its long-term objectives. Youth whose caregivers had originally provided consent and managed study visit appointments must consent for their own continuing follow-up once they reach the age of majority. This provides them with the opportunity to decline further participation in research. There are also special challenges inherent with following youth with chronic conditions into young adulthood, including fatigue after years of time-intensive clinical and research study visits, and the transition away from their paediatric or adolescent clinics, where their research visits often take place, into adult care clinics. ${ }^{22}$ Investigators conducting longterm research of young people with chronic, serious conditions, such as HIV infection, must consider the inevitable transition away from the strong support systems that often exist for these youth in paediatric care, but which may not exist in adult care systems, ${ }^{23-25}$ and the resulting effect on participants' willingness and ability to continue to participate in research as adults.

The long-term success of epidemiological studies of young adults will be built on flexible study designs that are less time intensive and more accommodating of participants' changing lifestyles than the clinic-based studies of their youth. A growing number of papers describe epidemiological studies that use computer-based and cellphone-based data collection strategies. ${ }^{26-31}$ In this paper, we describe the rationale for and development of AMP Up, a prospective cohort study that uses a flexible hybrid of online and in-person data collection to follow PHIV+ and PHEU youth as they enter adulthood. AMP Up was designed to address the primary scientific objective of defining the impact of HIV infection and antiretroviral therapy on young people with perinatal HIV infection as they transition into adulthood.

\section{METHODS AND ANALYSIS \\ Assessing feasibility of AMP Up protocol}

While national surveys suggest that most young adults in the USA access and frequently use the internet, ${ }^{32}$ socioeconomic disparities in access persist. ${ }^{33}$ Many PHACS participants come from socioeconomically disadvantaged backgrounds, ${ }^{2}$ and PHACS research site staff indicated that some study participants might have limited internet access. To determine whether PHACS participants would be able to complete the online surveys through which a large proportion of data in AMP Up would be collected, we conducted a feasibility study to evaluate the use of internet and mobile devices and willingness to participate in online research by youth and young adults seen at PHACS research clinics. The results of this anonymous survey are summarised in table 1 . Most individuals accessed the internet more than once a day, and the majority reported being willing to participate in online research.

We also considered the cognitive ability of PHACS participants to complete internet-based instruments in AMP Up. The AMP protocol collects sexual behaviour and substance use information via an audio computerassisted self-interview (ACASI), which is self-administered on a computer. If an ACASI is not completed at a given visit, the site records the reason. We compared full-scale IQ scores from the Wechsler Intelligence Scale for Children, Fourth Edition, ${ }^{34}$ for AMP youth unable to complete any ACASI due to cognitive limitations, and those who completed one or more ACASI. The results the ACASI review are summarised in table 2. The vast majority of AMP participants have completed at least one ACASI; the few participants who have not completed an ACASI because of cognitive limitations have significant impairments.

On the basis of the results of the internet use survey and the ACASI review, we expect that the vast majority of eligible AMP Up participants will be able to complete the online surveys independently. However, as we describe below, we have incorporated accommodations for those unable to do so.

\section{AMP Up study design}

AMP Up is a prospective cohort study which conducts yearly online data collection for the majority of self-reported information, annual medical chart abstraction and sexually transmitted infection (STI) specimen collection, and three in-person clinic visits at entry, year 3 and year 6 . Individual participants will be followed for a minimum of 6 years. The study is currently recruiting, with 305 individuals enrolled thus far. 
Table 1 Feasibility study results: responses from anonymous survey on internet and cellphone use by participants at PHACS clinical research sites, 2012-2013 $(\mathrm{N}=300)$

\begin{tabular}{|c|c|}
\hline Characteristic & $\mathbf{N}(\%)$ \\
\hline Age range & $16-27$ years \\
\hline Female sex & $144(48.0)$ \\
\hline African-American race & $212(70.7)$ \\
\hline Hispanic ethnicity & $70(23.3)$ \\
\hline PHACS participants & $134(44.7)$ \\
\hline \multicolumn{2}{|l|}{ Internet frequency in past 3 months } \\
\hline >once a day & $225(75.7)$ \\
\hline$<$ once a day/ $\geq$ once a week & $56(18.9)$ \\
\hline <once a week/never ${ }^{\star}$ & $16(5.4)$ \\
\hline \multicolumn{2}{|l|}{ Type of internet at home } \\
\hline DSL/cable & $76(25.3)$ \\
\hline Dial up & $1(0.3)$ \\
\hline Wireless/3G/4G & $125(41.8)$ \\
\hline Don't know & $43(14.3)$ \\
\hline No internet at home & $30(10.0)$ \\
\hline Missing & $25(8.3)$ \\
\hline \multicolumn{2}{|l|}{ Type of cellphone } \\
\hline Smart phone & $240(80.1)$ \\
\hline Phone that can send/receive texts & $26(8.8)$ \\
\hline No phone/cellphone doesn't text & $34(11.1)$ \\
\hline \multicolumn{2}{|l|}{ Cellphone with unlimited internet } \\
\hline Yes & 207 (69.3) \\
\hline No & $52(17.6)$ \\
\hline Don't know & $6(2.0)$ \\
\hline Cellphone doesn't access internet & $6(2.0)$ \\
\hline No cellphone & $28(9.1)$ \\
\hline \multicolumn{2}{|c|}{ Willingness to participate in online research $\dagger$} \\
\hline $\begin{array}{l}\text { Talk on cellphone about health or other } \\
\text { parts of life }\end{array}$ & $199(66.5)$ \\
\hline $\begin{array}{l}\text { Go online to answer questions about } \\
\text { health or other parts of life }\end{array}$ & $230(77.7)$ \\
\hline $\begin{array}{l}\text { Go to laboratory instead of clinic for blood } \\
\text { draw and other medical tests }\end{array}$ & $190(64.0)$ \\
\hline $\begin{array}{l}\text { Except where indicated, missing responses to ind } \\
\text { range from } 0 \text { to } 4 \text {. } \\
\text { "Two respondents reported no use of internet in } p\end{array}$ & $\begin{array}{l}\text { dual questions } \\
\text { t } 3 \text { months. } \\
\text { would'. }\end{array}$ \\
\hline
\end{tabular}

\section{Study population and inclusion criteria}

The eligible study population for AMP Up includes all PHIV+ and PHEU young adults 18 years of age and older previously followed in AMP (including those lost to follow-up in AMP), along with other PHIV+ young adults with available lifetime history of ARV use, CD4 and HIV-1 RNA, and major medical diagnoses. Perinatal HIV status must be documented in the patient's medical chart. All eligible participants are recruited from one of 14 clinical research sites across the USA including Puerto Rico. Enrolment opened in April 2014 to former AMP participants, and in May 2015 to other PHIV+ individuals previously involved in non-PHACS research protocols at the clinical research sites. The targeted enrolment goal is 600 PHIV+ and 250 PHEU young adults.
Table 2 Feasibility study results: ACASI completion and cognitive status of PHACS AMP participants

\begin{tabular}{|c|c|c|c|}
\hline $\begin{array}{l}\text { ACASI } \\
\text { completion } \\
\text { status }\end{array}$ & N (\%) & $\begin{array}{l}\text { Full scale IQ } \\
\text { score, mean } \\
(\mathrm{SD})^{\star}\end{array}$ & p Valuet \\
\hline $\begin{array}{l}\text { ACASI } \\
\text { mpleted due to } \\
\text { gnitive } \\
\text { itations }\end{array}$ & & $\ddagger$ & T \\
\hline $\begin{array}{l}\text { one ACASI } \\
\text { ompleted }\end{array}$ & $92(97.4)$ & 86. & \\
\hline \multicolumn{4}{|c|}{$\begin{array}{l}\text { *Full scale IQ score from Wechsler Intelligence Scale for Children, } \\
\text { Fourth Edition (WISC-IV). } \\
\text { †From t-test. } \\
\text { fDue to missing WISC-IV, N for those without ACASI=12, N for } \\
\text { ACASI completed=576. } \\
\text { ACASI, audio computer-assisted self-interview; AMP, Adolescent } \\
\text { Master Protocol; PHACS, Pediatric HIV/AIDS Cohort Study. }\end{array}$} \\
\hline
\end{tabular}

\section{Evaluations and data collection methods}

The AMP Up protocol is measuring outcomes from a wide range of domains, including: transition to adult clinical care; employment and educational achievement; retention in healthcare; HIV end-organ disease outcomes (renal, hepatic, cardiac, pulmonary and peripheral and central nervous systems) and HIV-associated malignancies; metabolic complications (dyslipidemia, hypertension, insulin resistance, hyperlactataemia, excess adiposity and fat redistribution); STIs; reproductive health; mental health and neurocognitive functioning; adherence to antiretroviral treatment (ART); sexual behaviour and substance use; and hearing and language impairments. The frequency of outcome and covariate measurement and data collection methods are summarised in table 3; the specific information obtained with each method of data collection is described in detail below.

\section{Data collection methods}

\section{Medical chart abstraction}

Medical and neuropsychological diagnoses, ARVs and other medications, reproductive history, laboratory measures, height, weight and blood pressure are abstracted from participants' medical records.

\section{In-person clinic visits}

Physical examinations, laboratory tests, intervieweradministered mental health evaluations (Client Diagnosis Questionnaire; ${ }^{35}$ Centers for Epidemiologic Studies Short Depression Scale ${ }^{36}$ ), and collection of repository storage specimens (blood, saliva, urine, vaginal swabs) are conducted. Cognitive function, hearing, emotional health and friendship are assessed via the online NIH Toolbox for the Assessment of Neurological and Behavioral Function (NIH Toolbox), ${ }^{37}$ and language is assessed with the interview-administered Clinical Evaluation of Language Fundamentals, Fourth Edition. ${ }^{38}$ Physical activity is evaluated with the online Block Physical Activity Screener. ${ }^{39}$ 
Table 3 Schedule of evaluations for the PHACS AMP Up study

\begin{tabular}{|c|c|c|c|c|c|c|c|c|}
\hline \multirow[b]{2}{*}{ Assessment } & \multirow[b]{2}{*}{$\begin{array}{l}\text { Data collection } \\
\text { method }\end{array}$} & \multicolumn{7}{|l|}{ Study visit } \\
\hline & & $\begin{array}{l}\text { Entry } \\
\text { (in-clinic) }\end{array}$ & Year 1 & Year 2 & $\begin{array}{l}\text { Year } 3 \\
\text { (in-clinic) }\end{array}$ & Year 4 & Year 5 & $\begin{array}{l}\text { Year } 6 \\
\text { (in-clinic) }\end{array}$ \\
\hline \multicolumn{9}{|c|}{ Demographic and psychosocial factors } \\
\hline $\begin{array}{l}\text { Education, employment, } \\
\text { housing }\end{array}$ & Online survey & $\sqrt{ }$ & $\sqrt{ }$ & $\sqrt{ }$ & $\sqrt{ }$ & $\sqrt{ }$ & $\sqrt{ }$ & $\sqrt{ }$ \\
\hline $\begin{array}{l}\text { Healthcare usage, } \\
\text { transition to adult clinical } \\
\text { care }\end{array}$ & Online survey & $\sqrt{ }$ & $\sqrt{ }$ & $\sqrt{ }$ & $\sqrt{ }$ & $\sqrt{ }$ & $\sqrt{ }$ & $\sqrt{ }$ \\
\hline $\begin{array}{l}\text { Social support, } \\
\text { friendships, self-efficacy }\end{array}$ & Online evaluation & $\sqrt{ }$ & & & $\sqrt{ }$ & & & $\sqrt{ }$ \\
\hline Quality of life & Online survey & $\sqrt{ }$ & $\sqrt{ }$ & $\sqrt{ }$ & $\sqrt{ }$ & $\sqrt{ }$ & $\sqrt{ }$ & $\sqrt{ }$ \\
\hline Physical activity & Online evaluation & $\sqrt{ }$ & & & $\sqrt{ }$ & & & $\sqrt{ }$ \\
\hline Nutrition & Online survey & & $\sqrt{ }$ & $\sqrt{ }$ & $\sqrt{ }$ & $\sqrt{ }$ & $\sqrt{ }$ & $\sqrt{ }$ \\
\hline \multicolumn{9}{|c|}{ Medical and neuropsychological factors } \\
\hline Diagnoses* $^{*}$ & Chart abstraction & $\sqrt{ }$ & $\sqrt{ }$ & $\sqrt{ }$ & $\sqrt{ }$ & $\sqrt{ }$ & $\sqrt{ }$ & $\sqrt{ }$ \\
\hline Medications & Chart abstraction & $\sqrt{ }$ & $\sqrt{ }$ & $\sqrt{ }$ & $\sqrt{ }$ & $\sqrt{ }$ & $\sqrt{ }$ & $\sqrt{ }$ \\
\hline $\begin{array}{l}\text { Height, weight, blood } \\
\text { pressure* }\end{array}$ & Chart abstraction & $\sqrt{ }$ & $\sqrt{ }$ & $\sqrt{ }$ & $\sqrt{ }$ & $\sqrt{ }$ & $\sqrt{ }$ & $\sqrt{ }$ \\
\hline Body measurements & Clinic visit & $\sqrt{ }$ & & & $\sqrt{ }$ & & & $\sqrt{ }$ \\
\hline Reproductive health & Online survey & $\sqrt{ }$ & $\sqrt{ }$ & $\sqrt{ }$ & $\sqrt{ }$ & $\sqrt{ }$ & $\sqrt{ }$ & $\sqrt{ }$ \\
\hline $\begin{array}{l}\text { Neurocognitive } \\
\text { functioning }\end{array}$ & Online evaluation & $\sqrt{ }$ & & & $\sqrt{ }$ & & & $\sqrt{ }$ \\
\hline $\begin{array}{l}\text { Mental health: depression } \\
\text { screen }\end{array}$ & Interview & $\sqrt{ }$ & & & $\sqrt{ }$ & & & $\sqrt{ }$ \\
\hline $\begin{array}{l}\text { Mental health: psychiatric } \\
\text { diagnoses }\end{array}$ & Interview & & & & $\sqrt{ }$ & & & $\sqrt{ }$ \\
\hline Hearing & Online evaluation & $\sqrt{ }$ & & & $\sqrt{ }$ & & & $\sqrt{ }$ \\
\hline Language & Interview & $\sqrt{ }$ & & & & & & \\
\hline $\begin{array}{l}\text { Laboratories done for } \\
\text { clinical care }\end{array}$ & Chart abstraction & $\sqrt{ }$ & $\sqrt{ }$ & $\sqrt{ }$ & $\sqrt{ }$ & $\sqrt{ }$ & $\sqrt{ }$ & $\sqrt{ }$ \\
\hline Laboratory biomarkers $†$ & Clinic visit & $\sqrt{ }$ & & & $\sqrt{ }$ & & & $\sqrt{ }$ \\
\hline $\begin{array}{l}\text { Sexually transmitted } \\
\text { infections }\end{array}$ & Self-collection & $\sqrt{ }$ & $\sqrt{ }$ & $\sqrt{ }$ & $\sqrt{ }$ & $\sqrt{ }$ & $\sqrt{ }$ & $\sqrt{ }$ \\
\hline $\begin{array}{l}\text { Repository samples } \ddagger \\
\text { Behavioural factors }\end{array}$ & Clinic visit & $\sqrt{ }$ & & & $\sqrt{ }$ & & & $\sqrt{ }$ \\
\hline Sexual behaviour & Online survey & $\sqrt{ }$ & $\sqrt{ }$ & $\sqrt{ }$ & $\sqrt{ }$ & $\sqrt{ }$ & $\sqrt{ }$ & $\sqrt{ }$ \\
\hline Substance use & Online survey & $\sqrt{ }$ & $\sqrt{ }$ & $\sqrt{ }$ & $\sqrt{ }$ & $\sqrt{ }$ & $\sqrt{ }$ & $\sqrt{ }$ \\
\hline ART adherence & Online survey & $\sqrt{ }$ & $\sqrt{ }$ & $\sqrt{ }$ & $\sqrt{ }$ & $\sqrt{ }$ & $\sqrt{ }$ & $\sqrt{ }$ \\
\hline
\end{tabular}

*Pregnancies, fractures, hearing problems, height and weight are also self-reported in annual online surveys.

†Venous lactate/pyruvate, renal biomarkers, cardiac biomarkers, fasting lipids, glucose, insulin.

$\ddagger$ Serum and plasma (EDTA and heparin), PBMC, throat wash/gargle, saliva, urine, vaginal swab.

AMP, Adolescent Master Protocol; EDTA, ethylenediamine tetraacetic acid; PBMC, peripheral blood mononuclear cells; PHACS, Pediatric HIVIAIDS Cohort Study.

\section{Self-collection of STI specimens}

For participants without Neisseria gonorrhoeae and Chlamydia trachomatis testing results available in medical records during the 12 months prior to a study visit, urine and vaginal swabs are self-collected for STI testing at the local clinical laboratory. Additional urine and swab samples are taken and sent to repository for further STI testing at a central research laboratory. At in-person clinic visits, samples are self-collected at the clinic, and at other time points are self-collected either at the clinic or at home.

\section{Online survey}

The AMP Up online survey consists of a series of short modules with questions on the following topics: employment/education/housing, healthcare, quality of life, sexual behaviours, reproductive health, substance use and nutrition; and for PHIV+ participants, adherence to ART and transition to adult clinical care. The survey also includes an acceptability question asking if participants would prefer to take the survey at home, in the clinic, or elsewhere, and a test question to assess how closely participants read the survey instructions. The survey ends with optional questions to assess participants' acceptability of the instrument.

Input from members of the PHACS Young Adult Community Advisory Board (YACAB) emphasised strong preference for short surveys that did not repeat questions that were answered in earlier surveys. We therefore 
developed surveys that can be completed at one or multiple sittings. Skip patterns are embedded within and also between surveys completed at different time points, allowing follow-up surveys to be tailored to each participant based on their responses to previous surveys.

Participants can opt to complete surveys at their study clinic or offsite. For individuals with cognitive limitations who cannot respond to survey questions on their own, we developed a shorter online instrument consisting of a subset of the modules (employment/education/ housing, healthcare usage, medication adherence and transition to adult HIV care) that can be completed by their caregiver. Alternatively, participants who can respond on their own but cannot self-administer the online instrument can complete a similar, intervieweradministered online survey that also includes the quality of life module.

While study clinics contact participants each year on the anniversary of their entry visit to remind them to complete their surveys, participants are able to complete the surveys at any point prior to the next entry visit anniversary. On completion, participants have the choice of a US\$75 virtual or physical gift card. This remuneration is provided in addition to funds provided by the clinical site to compensate for food, travel or participation in other study activities.

The online surveys were piloted by research and clinic staff, YACAB members, and other young adult volunteers from outside of PHACS. Surveys are thoroughly tested prior to implementation to ensure the proper placement and function of skip patterns. Responses to the surveys are regularly reviewed to allow for modification of potentially unclear questions.

\section{Training and implementation}

Given the novelty of internet-based instruments and remote data collection to PHACS staff and participants, we created written instructions to guide them through important study processes, including study registration, specimen sample collection for STI testing, registration and navigation of the NIH Toolbox, and organisation of the entry visit. The importance of the in-person entry visit is emphasised in the instructions, as it provides initial administration of the online survey in a familiar setting, and preparation for the year 1 follow-up visit where the first instance of remote data collection may take place.

\section{Research sites' role in data collection and participant engagement}

Since staff at the paediatric/adolescent clinics are often an important part of participants' systems of support, research site staff have emphasised that their relationship with participants should be maintained during this potentially turbulent period. The AMP Up protocol was therefore designed with flexibility in terms of the research sites' role in data collection and participant engagement, allowing participants to determine a comfortable pace by which to transition from clinicbased research visits to remote online data collection. Initially, AMP Up site staff will be integrally involved with all participants through study enrolment and completion of entry visits. For a certain subgroup of participants, this level of site involvement will continue throughout the duration of the protocol due to personal preference or because of lack of internet access or cognitive limitations. However, it is anticipated that the ability and willingness of most young adults to return to the clinic will diminish and eventually end due to competing priorities; therefore, accommodations have been incorporated to allow for their continued study participation entirely off-site, through chart abstraction, online data collection and mailed-in specimen collection.

\section{Sample size}

Our targeted sample size of 600 PHIV+ and 250 PHEU young adults will allow us to address AMP Up's primary scientific objective of defining the impact of HIV infection and antiretroviral therapy on young adults with perinatal HIV infection as they transition into adulthood. In the context of this study, we will be measuring many targeted outcomes, some continuous (eg, neurocognitive functioning defined as full-scale IQ score) and some binary (insulin resistance, retention in adult clinical care). For comparisons between PHIV+ and PHEU young adults, the target sample size of 600 perinatally infected versus 250 uninfected participants for the AMP Up study provides $80 \%$ power to detect a difference in means for a continuous outcome of 0.211 standard deviations (SDs) based on a two-sample t-test (assuming normality holds), and 0.216 based on a non-parametric Wilcoxon rank sum test (for skewed outcomes) at $\alpha=0.05$. For example, if we were comparing the full-scale IQ scores of HIV-infected versus uninfected participants and assumed a SD of 15 points, we could detect a difference of 3.16 points or more in the mean IQs. If we allow for possible loss to follow-up or incomplete/missing assessments of $4 \%$ per year for infected and $6 \%$ per year for uninfected participants for 3 years after enrolment into AMP Up is completed, then an adjusted sample size of 530 HIV-infected versus 208 HIV-uninfected participants provides $80 \%$ power to detect a difference of 0.229 SDs, or 3.44 points in mean IQ scores. Once we adjust for potential confounders, we will typically lose some power, so the minimum detectable difference will usually increase.

For binary outcomes, the minimum detectable odds ratios (ORs) we can detect based on comparing HIV-infected versus uninfected participants, at $80 \%$ power with a 0.05 significance level, ranges from 1.57 to 2.64 depending on underlying rate of the event in the comparison (PHEU) cohort, which we have varied in these sample size calculations to be between $4 \%$ and $30 \%$. For simplicity here, it is assumed that the event rate is higher in the HIV-infected group than the PHEU group. 
For comparisons within PHIV+ young adults we may wish, for example, to compare those PHIV+ young adults who initiated ART before age 5 years versus those who initiated ART at a later age. The detectable differences in means relative to the SD that can be detected assuming 530 PHIV+ participants (under an assumption of $4 \%$ loss per year) when two subgroups of the PHIV+ participants are being compared ranges from 0.24 to 0.41 , depending on the sample percentages in each subgroup. When comparing proportions with events between two subgroups, the minimum detectable ORs range from 1.63 to 4.01 depending on the percentages in each subgroup.

\section{Statistical analyses}

Participants' baseline characteristics (including demographics and clinical characteristics) will be described using frequencies for categorical variables and means, medians, SDs and IQR for continuous variables. Incidence estimates for outcomes will be calculated under a Poisson distribution based on participant-years of follow-up.

When evaluating associations between the many exposures and outcomes of interest in this study, multivariable regression analyses will be conducted adjusting for potential confounding factors. For cross-sectional associations, logistic and linear regression models will be used. Cox proportional hazards models will be used for factors associated with incidence of commonly occurring outcomes, and Poisson regression for incidence of outcomes that are observed to occur less frequently. For evaluation of factors associated with changes in outcomes over time, generalised estimating equation, or mixed effects models, will be employed. Effect modification of associations by specific covariates will be evaluated by adding interaction terms to the multivariable regression models. A probability level of 0.05 will be used for all analyses.

All analyses will be conducted using SAS V.9.2 (SAS Institute Inc, Cary, North Carolina, USA).

\section{ETHICS AND DISSEMINATION}

The AMP Up protocol was approved by the Harvard T.H. Chan School of Public Health's Institutional Review Board (IRB) as well as the IRBs at the 14 participating sites. Participants provide written informed consent; for cognitively impaired individuals with legally authorised representatives, legal guardian permission and participant assent is obtained.

To protect participant confidentiality, in addition to an anonymised Participant Identifier assigned to all PHACS participants, AMP Up participants are assigned a Personal Identification Number and Keyword to enter and submit online survey data. Written instructions were also developed for participants to provide steps to maintain their privacy when participating in web-based research. DatStat, ${ }^{40}$ which hosts the AMP Up online surveys, maintains stringent levels of encryption and data storage that exceed both industry standards for internet security and IRB standards for the protection of research participants and electronic records. DatStat's technology platform, including servers, databases, and web presences, employs multiple layers of security features to protect research participants and their data.

\section{Dissemination}

The findings from this study will be disseminated through peer-reviewed journals, national and international conference presentations, and to study participants through participant summaries that are presented in YACAB newsletters and in a recently-developed participant website.

\section{DISCUSSION}

Many PHACS participants have a strong connection with their clinical research site, as expressed by YACAB members and site staff. This connection may minimise the participation and retention issues experienced by other epidemiological studies recruiting and following participants entirely online. ${ }^{41}$ The long-term success of AMP Up will be tested as more participants relocate, attend college, transfer their clinical care to adult clinics (a process typically completed by age 25 years), or take on work and family responsibilities that preclude them from completing study assessments in the clinic at which they enrolled in AMP Up. While our initial success may hinge on the strong connections participants have with their sites, in order to successfully transition them to remote visits, we will need to encourage direct connections between participants and the PHACS study itself. For this reason, we are taking steps to strengthen the association AMP Up participants have with PHACS. This began with the involvement of AMP participants in the development of AMP Up, and has continued with the development of the YACAB and of the participant website, through which participants link to their online surveys, access information on health, education and housing opportunities, and connect with other study members.

Given it will be important for the majority of AMP participants to successfully enrol into AMP Up as they reach 18 years of age, we are beginning to examine enrolment trends and reasons for not enrolling. Additional challenges in recruitment and study retention will arise when AMP Up opens enrolment to PHIV+ young adults not previously involved in research protocols at our research clinics. We are exploring methods to offer web-based informed consent forms, enrolment and medical record release authorisations for these potential participants. The close connection our participants experience with their clinic sites also emphasises an important limitation of our study. Since we are enrolling young adults through these clinical sites, our participants may be more likely to be adherent to clinical care and supportive services; young adults who are non-adherent or lost to care may not be well represented in our cohort.

In some aspects, the first generation of perinatally HIV-infected youth is unique. Many of these young 
people were born before the advent of effective antiretroviral therapy and were not expected to live past childhood. This expectation may have profound consequences for their transition into young adulthood, a transition which is of particular research interest both domestically and internationally. To potentiate the opportunity to collaboratively study transition, we have shared the AMP Up protocol and online survey instrument with other global networks whose aims include an examination of the transition into adult clinical care for young people infected with HIV, including the International Epidemiologic Databases to Evaluate AIDS $^{42}$ and EuroCoord. ${ }^{43}$ The design of AMP Up, which allows flexibility in the transition from in-person to remote data collection, can also enhance long-term research possibilities for a broader set of studies. Investigations undertaking long-term follow-up of young adults with chronic conditions, including birth or early childhood cohorts, or more generally, any epidemiological studies for which long-term success is contingent on the ability to retain participants' interest and long-term commitment, can potentially benefit from adopting this study design.

\author{
Author affiliations \\ ${ }^{1}$ Department of Epidemiology, Harvard T.H. Chan School of Public Health, \\ Boston, Massachusetts, USA \\ ${ }^{2}$ Center for Biostatistics in AIDS Research, Harvard T.H. Chan School of \\ Public Health, Boston, Massachusetts, USA \\ ${ }^{3}$ Frontier Science and Technology Research Foundation, Inc, Amherst \\ New York, USA \\ ${ }^{4}$ Division of AIDS Research, National Institute of Mental Health, National \\ Institutes of Health, Bethesda, Maryland, USA \\ ${ }^{5}$ Maternal and Pediatric Infectious Disease Branch, Eunice Kennedy Shriver \\ National Institute of Child Health and Human Development, National Institutes \\ of Health, Bethesda, Maryland, USA \\ ${ }^{6}$ Department of Pediatric Infectious Diseases, University of Colorado, \\ Children's Hospital Colorado, Aurora, Colorado, USA \\ ${ }^{7}$ Department of Health Behavior and Health Education, University of Michigan \\ School of Public Health, Ann Arbor, Michigan, USA \\ ${ }^{8}$ Department of Pediatrics, Tulane University School of Medicine, New \\ Orleans, Louisiana, USA \\ ${ }^{9}$ Children's Diagnostic \& Treatment Center, Inc., Fort Lauderdale, Florida, USA
}

Acknowledgements The authors thank the children and families for their participation in PHACS, and the individuals and institutions involved in the conduct of PHACS. The study was supported by the Eunice Kennedy Shriver National Institute of Child Health and Human Development with cofunding from the National Institute on Drug Abuse, the National Institute of Allergy and Infectious Diseases, the Office of AIDS Research, the National Institute of Mental Health, the National Institute of Neurological Disorders and Stroke, the National Institute on Deafness and Other Communication Disorders, the National Heart Lung and Blood Institute, the National Institute of Dental and Craniofacial Research, and the National Institute on Alcohol Abuse and Alcoholism, through cooperative agreements with the Harvard T.H. Chan School of Public Health (HD052102) (Principal Investigator: George Seage; Project Director: Julie Alperen) and the Tulane University School of Medicine (HD052104) (Principal Investigator: Russell Van Dyke; Co-Principal Investigators: Kenneth Rich, Ellen Chadwick; Project Director: Patrick Davis). Data management services were provided by Frontier Science and Technology Research Foundation (PI: Suzanne Siminski), and regulatory services and logistical support were provided by Westat, Inc (PI: Julie Davidson). The following institutions, clinical site investigators and staff participated in conducting PHACS AMP and AMP Up in 2015, in alphabetical order: Ann \& Robert H Lurie Children's Hospital of Chicago: Ram Yogev, Margaret Ann Sanders, Kathleen Malee, Scott Hunter; Baylor College of Medicine: William Shearer, Mary Paul, Norma Cooper, Lynnette Harris; Bronx
Lebanon Hospital Center: Murli Purswani, Mahboobullah Baig, Anna Cintron; Children's Diagnostic \& Treatment Center: Ana Puga, Sandra Navarro, Patricia Garvie, James Blood; Children's Hospital, Boston: Sandra Burchett, Nancy Karthas, Betsy Kammerer; Jacobi Medical Center: Andrew Wiznia, Marlene Burey, Molly Nozyce; Rutgers—New Jersey Medical School: Arry Dieudonne, Linda Bettica, Susan Adubato; St Christopher's Hospital for Children: Janet Chen, Maria Garcia Bulkley, Latreaca Ivey, Mitzie Grant; St Jude Children's Research Hospital: Katherine Knapp, Kim Allison, Megan Wilkins; San Juan Hospital/Department of Pediatrics: Midnela Acevedo-Flores, Heida Rios, Vivian Olivera; Tulane University Health Sciences Center: Margarita Silio, Medea Jones, Patricia Sirois; University of California, San Diego: Stephen Spector, Kim Norris, Sharon Nichols; University of Colorado Denver Health Sciences Center: Elizabeth McFarland, Alisa Katai, Jennifer Dunn, Suzanne Paul; University of Miami: Gwendolyn Scott, Patricia Bryan, Elizabeth Willen.

Collaborators This article is being submitted for the Pediatric HIV/AIDS Cohort Study.

Contributors Author KT was integrally involved in the conception and design of the protocol and drafting of the manuscript; authors GRS, RVD, RH and SMA were integrally involved in the conception and design of the protocol, reviewed the manuscript and provided revisions; author KP was involved in the design of the protocol and provided critical input on content and organisation of the paper; author JA was involved in the design and implementation of the protocol, wrote segments of the manuscript's Methods section and provided critical feedback on the manuscript in full; author DK was involved in the design of the protocol, reviewed the manuscript and provided important revisions; authors $\mathrm{KC}, \mathrm{SS}, \mathrm{MM}, \mathrm{AE}$ and $\mathrm{CB}$ were involved in the development of one or more of the protocol's data collection instruments, reviewed the manuscript and provided important revisions; authors JB and DQS provided important insight as to the structure and content of the online survey instrument, reviewed and revised the manuscript, and authors EB and AP participated in data acquisition and reviewed and revised the manuscript.

Funding The Pediatric HIV/AIDS Cohort Study (PHACS) was supported by the Eunice Kennedy Shriver National Institute of Child Health and Human Development with cofunding from the National Institute on Drug Abuse, the National Institute of Allergy and Infectious Diseases, the Office of AIDS Research, the National Institute of Mental Health, the National Institute of Neurological Disorders and Stroke, the National Institute on Deafness and Other Communication Disorders, the National Heart Lung and Blood Institute, the National Institute of Dental and Craniofacial Research, and the National Institute on Alcohol Abuse and Alcoholism, through cooperative agreements with the Harvard T.H. Chan School of Public Health (HD052102) and the Tulane University School of Medicine (HD052104). Note: The conclusions and opinions expressed in this article are those of the authors and do not necessarily reflect those of the National Institutes of Health or the US Department of Health and Human Services.

Competing interests None declared.

Provenance and peer review Not commissioned; externally peer reviewed.

Ethics approval Institutional Review Board (IRB) of the Harvard T.H. Chan School of Public Health; IRBs of the 14 participating sites.

Data sharing statement Researchers interested in collaborations or further information regarding the protocol are invited to visit the PHACS website https://phacsstudy.org.

Open Access This is an Open Access article distributed in accordance with the Creative Commons Attribution Non Commercial (CC BY-NC 4.0) license, which permits others to distribute, remix, adapt, build upon this work noncommercially, and license their derivative works on different terms, provided the original work is properly cited and the use is non-commercial. See: http:// creativecommons.org/licenses/by-nc/4.0/

\section{REFERENCES}

1. Jacobson DL, Patel K, Siberry GK, et al. Body fat distribution in perinatally HIV-infected and HIV-exposed but uninfected children in the era of highly active antiretroviral therapy: outcomes from the Pediatric HIV/AIDS Cohort Study. Am J Clin Nutr 2011;94:1485-95. 
2. Malee KM, Tassiopoulos K, Huo Y, et al. Mental health functioning among children and adolescents with perinatal HIV infection and perinatal HIV exposure. AIDS Care 2011;23:1533-44.

3. Miller TI, Borkowsky W, DiMeglio LA, et al. Metabolic abnormalities and viral replication are associated with biomarkers of vascular dysfunction in HIV-infected children. HIV Med 2012;13:264-75.

4. Patel K, Wang J, Jacobson DL, et al. Aggregate risk of cardiovascular disease among adolescents perinatally infected with the human immunodeficiency virus. Circulation 2014;129:1204-12.

5. Purswani M, Patel K, Kopp JB, et al. Tenofovir treatment duration predicts proteinuria in a multiethnic United States Cohort of children and adolescents with perinatal HIV-1 infection. Pediatr Infect Dis J 2013;32:495-500.

6. Rice ML, Buchanan AL, Siberry GK, et al. Language impairment in children perinatally infected with HIV compared to children who were HIV-exposed and uninfected. J Dev Behav Pediatr 2012;33:112-23.

7. Siberry GK, Patel K, Van Dyke RB, et al. CD4+ lymphocyte-based immunologic outcomes of perinatally HIV-infected children during antiretroviral therapy interruption. J Acquir Immune Defic Syndr 2011;57:223-9.

8. Tassiopoulos K, Moscicki AB, Mellins C, et al. Sexual risk behavior among youth with perinatal HIV infection in the United States: predictors and implications for intervention development. Clin Infect Dis 2013;56:283-90.

9. Torre P III, Zeldow B, Hoffman HJ, et al. Hearing loss in perinatally HIV-infected and HIV-exposed but uninfected children and adolescents. Pediatr Infect Dis J 2012;31:835-41.

10. Van Dyke RB, Patel K, Siberry GK, et al. Antiretroviral treatment of US children with perinatally acquired HIV infection: temporal changes in therapy between 1991 and 2009 and predictors of immunologic and virologic outcomes. J Acquir Immune Defic Syndr 2011;57:165-73.

11. Alperen J, Brummel S, Tassiopoulos K, et al. Prevalence of and risk factors for substance use among perinatally human immunodeficiency virus-infected and perinatally exposed but uninfected youth. J Adolesc Health 2014;54:341-9.

12. Williams PL, Abzug MJ, Jacobson DL, et al. Pubertal onset in children with perinatal HIV infection in the era of combination antiretroviral treatment. AIDS 2013;27:1959-70.

13. Mellins CA, Tassiopoulos $\mathrm{K}$, Malee $\mathrm{K}$, et al. Behavioral health risks in perinatally HIV-exposed youth: co-occurrence of sexual and drug use behavior, mental health problems, and nonadherence to antiretroviral treatment. AIDS Patient Care STDS 2011; 25:413-22.

14. Geffner ME, Patel K, Miller TL, et al. Factors associated with insulin resistance among children and adolescents perinatally infected with HIV-1 in the pediatric HIV/AIDS cohort study. Horm Res Paediatr 2011;76:386-91.

15. Lipshultz SE, Williams PL, Wilkinson JD, et al Cardiac status of children infected with human immunodeficiency virus who are receiving long-term combination antiretroviral therapy: results from the Adolescent Master Protocol of the Multicenter Pediatric HIV/ AIDS Cohort Study. JAMA Pediatr 2013;167:520-7.

16. The Pediatric HIV/AIDS Cohort Study (PHACS). https://phacsstudy. org/ (accessed Jan 2016).

17. DiMeglio LA, Wang J, Siberry GK, et al. Bone mineral density in children and adolescents with perinatal HIV infection. AIDS 2013;27:211-20.

18. Sohn $\mathrm{AH}$, Hazra R. The changing epidemiology of the global paediatric HIV epidemic: keeping track of perinatally HIV-infected adolescents. J Int AIDS Soc 2013;16:18555.

19. Mofenson LM, Cotton MF. The challenges of success: adolescents with perinatal HIV infection. J Int AIDS Soc 2013;16:18650.

20. Galea S, Tracy M. Participation rates in epidemiologic studies. Ann Epidemiol 2007;17:643-53.

21. Morton LM, Cahill J, Hartge P. Reporting participation in epidemiologic studies: a survey of practice. Am J Epidemiol 2006;163:197-203.
22. Callahan ST, Winitzer RF, Keenan P. Transition from pediatric to adult-oriented health care: a challenge for patients with chronic disease. Curr Opin Pediatr 2001;13:310-16.

23. Vander Stoep A, Beresford SA, Weiss NS, et al. Community-based study of the transition to adulthood for adolescents with psychiatric disorder. Am J Epidemiol 2000;152:352-62.

24. Fair CD, Sullivan K, Dizney R, et al. 'It's like losing a part of my family': transition expectations of adolescents living with perinatally acquired HIV and their guardians. AIDS Patient Care STDS 2012;26:423-9.

25. Committee On Pediatric Aids. Transitioning HIV-infected youth into adult health care. Pediatrics 2013;132:192-7.

26. Baer A, Saroiu S, Koutsky LA. Obtaining sensitive data through the Web: an example of design and methods. Epidemiology 2002;13:640-5.

27. Smith B, Smith TC, Gray GC, et al. When epidemiology meets the Internet: Web-based surveys in the Millennium Cohort Study. Am J Epidemiol 2007;166:1345-54.

28. Weisel CP, Weiss SH, Tasslimi A, et al. Development of a Web-based questionnaire to collect exposure and symptom data in children and adolescents with asthma. Ann Allergy Asthma Immuno 2008;100:112-19

29. Huntington A, Gilmour J, Schluter $P$, et al. The Internet as a research site: establishment of a web-based longitudinal study of the nursing and midwifery workforce in three countries. $J$ Adv Nurs 2009;65:1309-17.

30. Huybrechts KF, Mikkelsen EM, Christensen T, et al. A successful implementation of e-epidemiology: the Danish pregnancy planning study 'Snart-Gravid'. Eur J Epidemiol 2010;25:297-304.

31. van Gelder MM, Bretveld RW, Roeleveld N. Web-based questionnaires: the future in epidemiology? Am J Epidemiol 2010;172:1292-8

32. Lenhart A. Teen, Social Media and Technology Oveview 2015 2015. http://www.pewinternet.org/files/2015/04/PI_TeensandTech_ Update2015_0409151.pd (accessed Jan 2016).

33. File T, Camille R. Computer and Internet Use in the United States: 2013. American Community Survey Reports, ACS-28. Washington DC, 2014. https://www.census.gov/content/dam/ Census/library/publications/2014/acs/acs-28.pdf (accessed Dec 2015).

34. Wechsler D. The Wechsler Intelligence Scale for Children-Fourth Edition. San Antonio, TX: The Psychological Corporation; 2003.

35. Aidala A, Havens J, Mellins CA, et al. Development and validation of the Client Diagnostic Questionnaire (CDQ): a mental health screening tool for use in HIV/AIDS service settings. Psychol Health Med 2004:9:362-80.

36. Zhang W, O'Brien N, Forrest Jl, et al. Validating a shortened depression scale (10 item CES-D) among HIV-positive people in British Columbia, Canada. PLoS ONE 2012;7:e40793.

37. Gershon RC, Wagster MV, Hendrie HC, et al. NIH toolbox for assessment of neurological and behavioral function. Neurology 2013;80(Suppl 3):S2-6

38. Semel E, Wiig E, Secord W. Clinical evaluation of language fundamentals-4th edition. San Antonio, TX: The Psychological Corporation, 2003

39. NutritionQuest. Block Physical Activity Screener-Adults. https:// nutritionquest.com/assessment/list-of-questionnaires-and-screeners/ (accessed Sep 2015)

40. DatStat. DatStat. http://www.datstat.com (accessed Jan 2016)

41. Bajardi $P$, Paolotti D, Vespignani A, et al. Association between recruitment methods and attrition in Internet-based studies. PLoS ONE 2014:9:e114925.

42. International Epidemiologic Databases to Evaluate AIDS (IeDEA). http://www.iedea.org/ (accessed Jan 2016).

43. Enhancing clinical and epidemiological HIV research in Europe through cohort collaboration (EuroCoord). http://www.eurocoord.net/ (Accessed Jan 2016). 